\section{BRIDGING THE PLANNING EXECUTION GAP IN ROYAL COMMISSION HEALTH SERVICES PROGRAM YANBU (RCHSPY)}

Ammar Ashari, Mahmoud Radwhan, Fuad Alshoabi. Planning Section/Quality Management and Planning Department, Royal Commission Health Services Program

\subsection{6/bmjoq-2019-PSF.22}

Background The planning-execution gap is one of the main challenges facing any organization. According to the literature, the planning- execution gap is one of the leading causes of strategic and operational plan failure. Because of the complexity of healthcare, planning is more challenging. Inspired by Saudi Vision 2030 and guided by the National Transformation Program 2020, Royal Commission Health Services Program Yanbu (RCHSPY) made its Strategic and Operational Plan 2017-2020. The planning section identified the planning-execution gap as one of the main challenges impeding the progress of RCHSPY plans. This gap can result from many causes, such as ineffective communication, lack of measures and feedback, lack of collaboration, and weak alignment between strategic and operational plans. The planning section tried to study the gap by assessing the existing status and identifying the root causes in RCHSPY, then applying several tools and techniques in order to overcome this gap.

Methods RCHSPY aims to evaluate the involvement and awareness of all staff, particularly front liners and junior staff, about strategic and operational plans to ensure that they are fully oriented about the direction of the RCHSPY and that they feel their daily work is aligned with the direction of the RCHPSY strategic and operational plan. Surveys were carried out to assess the planning effectiveness and the planning-execution gap. The surveys asked staff about orientation, active participation, alignment, collaboration, and understanding of the initiatives and key performance indicators (KPIs). The survey results were supported by the analysis of 2017 and 2018 operational plan outcomes, as some significant findings have been observed.

Results The survey results showed that $50 \%$ of staff feel unsatisfied with their orientation, 34\% feel unsatisfied about their participation in RCHSPY plans, 27\% feel that their daily work is not aligned with RCHPSY plans, 50\% feel unsatisfied about collaboration with other departments to implement different initiatives, and 25\% replied that they never discussed RCHSPY in their departments. The 2017 and 2018 operational plan analysis of stumbling projects and initiatives showed four main challenges (4C): communication, collaboration, financial and managerial constraints, and connecting departmental KPIs to RCHSPY plans, which support the survey results.

Conclusion More effective communication methods are greatly required, such as interactive workshops and face-toface meetings, to enhance the involvement and empowerment of all staff in the planning process. Feedback should be delivered to all concerned stakeholders in the form of quarterly reports and KPI results should be discussed at all levels, particularly at the departmental level. Future studies about the planning-execution gap should be done to monitor progress.

\section{THE SUCCESSFUL JOURNEY OF THE FIRST A2LA ISO 15189:2012 MEDICAL LABORATORY IN THE KINGDOM OF SAUDI ARABIA}

Kaneez Zamir, Omar Qassas, Abdulaziz Johani, Abdulrahman Aboud. Department of Pathology and Laboratory Medicine, Prince Mohammed Bin Abdul Aziz Hospital - Al Madinah

\subsection{6/bmjoq-2019-PSF.23}

Background The aim was to improve the overall quality management system for the Department of Pathology and Laboratory Medicine by attaining international accreditation to A2LA ISO 15189 , in order to provide accurate, reliable, and timely results for excellent patient care and patient safety. The accreditation of laboratory medicine improves all processes in the laboratory, including reduction of errors in the pre-analytical, analytical, and post-analytical processes, facilitation of accurate and rapid diagnostics, participation in acceleration and efficiency of treatment, and stimulation of continuous improvement; therefore, the decision was taken to aim for A2LA ISO 15189 accreditation.

Methods

- Baseline assessment of the serology section was chosen as the model of implementation once successfully completing of all the identified gaps from the ISO 15189 standard.

- Development of project management action plan and agreed upon timeline:

- Accreditation Project Initiation 2015

- Accreditation Project Identification and Planning 2015-2016

- Accreditation Project Execution 2016-2017

- The ISO 15189 parameters were applied to the serology section and monitored by use of key performance indicators (KPIs).

- Accreditation Project control 2017

- The control was through performing internal audits and then taking corrective action with preventive action.

- Accreditation Project Closeout 2018

- The project was submitted for application to the American Association for Laboratory Accreditation ISO 15189.

Results Internal audits were performed from the baseline audit in 2015 to the final exit audit in 2018. The results for all the sections were as follows:

Chemistry: 2015 - 20\%; 2016 - 40\%; 2017 - 46\%; 2018 - 95\%. Hematology: 2015 - 51\%; 2016 - 60\%; 2017 - 62\%; $2018-91 \%$. Microbiology: 2015 - 19\%; 2016 - 40\%; 2017 - 46\%; 2018 - 85\%. Serology: 2015 - 52\%; 2016 - 65\%; 2017 - 83\%; 2018 - 95\%. Receiving OPD: 2015 - 39\%; 2016 - 52\%; 2017 - 70\%; 2018 - 90\%. Blood bank: 2015 - 20\%; 2016 - 50\%; 2017 - 70\%; 2018 - 83\%. Molecular biology: 2016 - 20\%; 2017 - 60\%; $2018-85 \%$.

Histopathology: 2015 - 15\%; 2016 - 30\%; 2017 - 60\%; 2018 - 89\%.

From the results, between 2016 and 2018, a dramatic increase in compliance was demonstrated, and therefore preparation for the final A2LA ISO 15189 audit was executed.

Conclusion An essential component of successful implementation of A2LA ISO 15189 was the coordination and rapport between management and staff. Well-trained and well-motivated laboratory staff were required to implement the system. The initial gap analysis and then the continuous monitoring through internal and external assessment provided invaluable 